\title{
Silver-Nanoparticle-Based Screen-Printing and Film Characterization of a Disposable, Dual-Band, Bandstop Filter on a Flexible Polyethylene Terephthalate Substrate
}

\author{
Kishor Kumar Adhikari, ${ }^{1}$ Younsu Jung, ${ }^{2}$ Hyejin Park, ${ }^{2}$ \\ Gyoujin Cho, ${ }^{2}$ and Nam-Young Kim ${ }^{1}$ \\ ${ }^{1}$ RFIC Center, Kwangwoon University, 447-1 Wolgye-dong, Nowon-ku, Seoul 139-701, Republic of Korea \\ ${ }^{2}$ Department of Printed Electronics Engineering, Sunchon National University, Maegok, Suncheon, \\ Jeonnam 540-742, Republic of Korea \\ Correspondence should be addressed to Nam-Young Kim; nykim@kw.ac.kr
}

Received 26 June 2015; Accepted 8 October 2015

Academic Editor: Luca Valentini

Copyright ( 2015 Kishor Kumar Adhikari et al. This is an open access article distributed under the Creative Commons Attribution License, which permits unrestricted use, distribution, and reproduction in any medium, provided the original work is properly cited.

\begin{abstract}
This paper presents a silver-nanoparticle-based, screen-printed, high-performance, dual-band, bandstop filter (DBBSF) on a flexible polyethylene terephthalate (PET) substrate. Using screen-printing techniques to process a highly viscous silver printing ink, highconductivity printed lines were implemented at a web transfer speed of $5 \mathrm{~m} / \mathrm{min}$. Characterized by X-ray diffraction (XRD), optical microscopy, atomic force microscopy (AFM), and scanning electron microscopy (SEM), the printed lines were shown to be characterized by smooth surfaces with a root mean square roughness of $7.986 \mathrm{~nm}$; a significantly higher thickness $(12.2 \mu \mathrm{m})$ than the skin depth; and a high conductivity of $2 \times 10^{7} \mathrm{~S} / \mathrm{m}$. These excellent printed line characteristics enabled the implementation of a high-selectivity DBBSF using shunt-connected uniform impedance resonators (UIRs). Additionally, the inductive loading effect of T-shaped stubs on the UIRs, which were analyzed using S-parameters based on lumped parameter calculations, was used to improve the return losses of the geometrically optimized DBBSF. The measured minimum return loss and maximum insertion loss of 28.26 and $1.58 \mathrm{~dB}$, respectively, at the central frequencies of 2.56 and $5.29 \mathrm{GHz}$ of a protocol screen-printed DBBSF demonstrated the excellent performance of the material and its significant potential for use in future cost-effective, flexible WiMax and WLAN applications.
\end{abstract}

\section{Introduction}

Screen-printing, which is also widely known as thick-film printing, is a promising candidate for creating high demand, environmentally friendly, light-weight, and cost-effective electronic products at low cost, high throughput, and high reproducibility [1-3]. Due to its numerous advantages, such as its high processing speed, high throughput, and high reproducibility, as well as its capacity to process highly viscous printing ink, this technique has been used in a diverse range of applications, including printed energy sources, diodes, resistors, sensors, microwave antennas, and filters $[4,5]$. Its capacity to work with several cheap substrates, such as paper, plastics, and glass, makes it suitable for creating light-weight, flexible, transparent, and disposable products $[6,7]$. However, using these substrates dictates low working temperatures; to meet this requirement, conductive inks with metal micro/nanoparticles have been widely researched and have been shown to be promising, because the increase in the ratio of surface area to volume with the reduction of the particles in volume significantly lowers the melting point of the particles compared with that of bulk materials [8]. As a consequence, several nanoparticles of noble metals, such as gold $(\mathrm{Au})$, silver $(\mathrm{Ag})$, platinum $(\mathrm{Pt})$, palladium $(\mathrm{Pd})$, or combinations of these metals, have been extensively investigated for the preparation of conductive pastes. However, Ag-particles-based ink is most widely used to manufacture conductive patterns in electronic devices due to its high stability in air and its highconductivity $[8,9]$. 
Currently, screen-printing-based implementations of radio-frequency, printed antennas and filters that are compact and highly selective are being investigated in many studies [10]. Various aspects such as the viscosity and surface tension of printing ink and the selection of an appropriate substrate and optimization of printing and sintering processes must be considered to successfully create a high-performance radio-frequency component. Kao et al. presented a silver-nanoparticle-colloidal-solution-based, inkjet-printed, radio-frequency bandpass filter on a liquid crystal polymer (LCP) substrate. Increasing the printed line conductivity and optimization of the line thickness with a uniform width were primarily considered to improve the performance of the filters. A hairpin bandpass filter [11], an interdigital coupled-line-based bandpass filter [12], and a third-order, dual-behavior resonator (DBR) bandpass filter [13] were inkjet-printed onto an LCP substrate. However, the large insertion losses indicated by the measurement results, which degraded the selective performance of the filters, were most likely caused by an insufficient conductivity of the printed lines. Therefore, increasing the conductivity of the printed lines to create a high-performance microwave filter is still an ongoing challenge.

In this study, we propose using Ag-nanoparticle-based screen-printing technique to create a high-performance, dual-band bandstop filter (DBBSF) on a flexible PET substrate. High-conductivity printed lines to obtain high return and low insertion losses that are indicative of excellent inband and out-of-band selectivity were the primary objectives of this study. The Ag-nanoparticle-based ink used in this study was formulated to have a high viscosity that was suitable for screen-printing of high-conductivity, uniform filter lines with smooth surfaces. Screen-printed Ag line characterization, which was performed by optical microscopy, scanning electron microscopy (SEM), and atomic force microscopy (AFM), indicated that the printed lines exhibited uniform widths with a thickness (e.g., $12.2 \mu \mathrm{m}$ ) significantly larger than the skin depth $(\delta)$ over nearly the entire operating frequency band; these analyses also showed that the lines had smooth surfaces with root mean square (RMS) value of roughness equal to $7.986 \mathrm{~nm}$ and a high-conductivity of $2 \times 10^{7} \mathrm{~S} / \mathrm{m}$. All of these excellent line characteristics allowed the creation of high-performance DBBSF with excellent selectivity and design flexibility based on stub-loaded, shunt-connected, uniform-impedance resonators (UIRs). A measured minimum return loss of $28.26 \mathrm{~dB}$ and a maximum insertion loss of $1.58 \mathrm{~dB}$ in the stop bands demonstrated the excellent selectivity of a protocol DBBSF operating at central frequencies of 2.56 and $5.29 \mathrm{GHz}$; and, therefore, the proposed process is a suitable candidate for use in low-cost WiMax and WLAN applications.

\section{Methods and Materials}

2.1. Layout Design, Simulation, and Screen-Printing. The 3D layout of the proposed DBBSF, which is shown in Figure 1, consists of two shunt-connected resonators with impedance and characteristic electrical lengths of $Z_{1}\left(\theta_{1}\right), Z_{2}\left(\theta_{2}\right)$, and

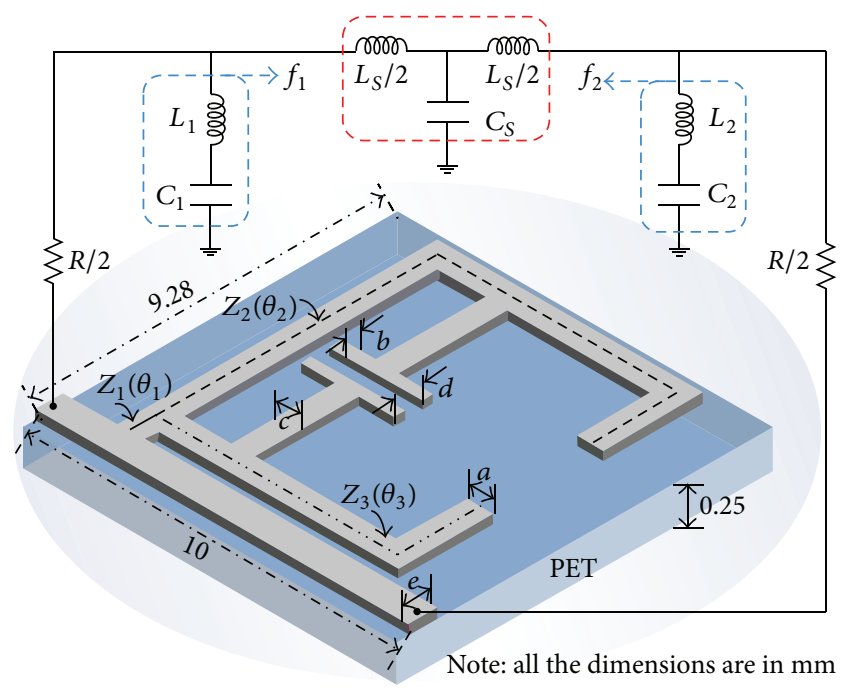

$\begin{array}{ll}a: 0.7 & d: 0.76 \\ \text { b: } 0.46 & \text { e: } 1 \\ c: 0.6 & \end{array}$

Figure 1: 3D layout and equivalent circuit of the proposed Agnanoparticle-based screen-printed DBBSF on PET substrate using T-shaped stub-loaded, shunt-connected UIRs.

$Z_{3}\left(\theta_{3}\right)$. Using $Z_{1}=Z_{2}=Z_{3}=Z$, the input impedance $\left(Z_{\text {in }}\right)$ of the proposed shunt-connected UIRs can be expressed as [14]

$$
Z_{\text {in }}=j Z \frac{\tan \theta_{1}\left(\tan \theta_{2}+\tan \theta_{3}\right)-1}{\tan \theta_{1}+\tan \theta_{2}+\tan \theta_{3}} .
$$

The solution of $Z_{\text {in }}=0$, which corresponds to the lower resonant frequency $\left(f_{1}\right)$ and the upper resonant frequency $\left(f_{2}\right)$ of the proposed shunt-connected UIRs, indicates that the frequency ratio $\left(f_{2} / f_{1}\right)$ can be varied within a wide range and the required resonant frequencies can be obtained by varying and optimizing the length ratios of $\theta_{1} /\left(\theta_{1}+\theta_{2}\right)$ and $\theta_{3} /\left(\theta_{1}+\right.$ $\theta_{2}$ ), respectively. $f_{2}$ can be independently varied by shifting to a lower UIR position along the impedance line of the upper UIR $[15,16]$. To verify the application of the proposed shuntconnected UIRs in the DBBSF, a protocol filter was designed on a PET substrate with a dielectric constant $\varepsilon_{r}=3$, a loss tangent $\delta=0.0147$, and a thickness $h=250 \mu \mathrm{m}$. Figure 2 also shows the equivalent circuit of the proposed DBBSF. $R$ represents the combined resistive loss of the tuned circuits $L_{1} C_{1}$ and $L_{2} C_{2}$ that correspond to the lower and upper UIRs, which resonate at $f_{1}$ and $f_{2}$, respectively; $L_{S}$ and $C_{S}$ represent the net inductive and capacitive effects of the T-shaped stubloads, respectively. The designed filter was simulated and optimized using a full-wave EM simulator, Sonnet, and the optimized dimensional parameters of UIRs, which exhibited resonant frequencies of 2.9 and $5.65 \mathrm{GHz}$, as shown in the simulated results of Figure 3 (a) are $Z=76.58 \Omega, \theta_{1}=4.63^{\circ}$, $\theta_{2}=28.6^{\circ}$, and $\theta_{3}=23.2^{\circ}$. To obtain DBBSF with improved return losses, the stub with the optimized dimensions of $Z_{s 1}=33.7 \Omega, Z_{s 2}=82.78 \Omega, \theta_{s 1}=12.55^{\circ}$, and $\theta_{s 2}=1.35^{\circ}$ was loaded onto the UIRs. 

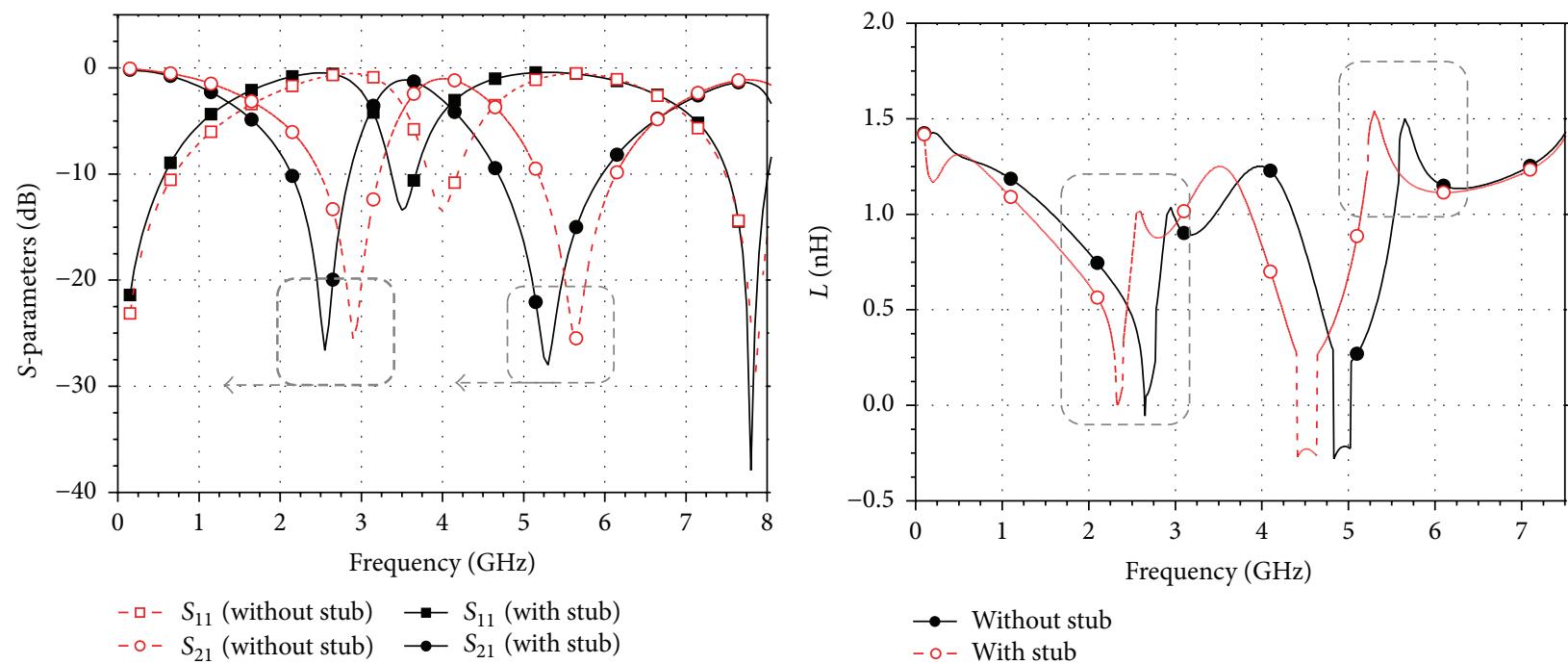

$-\square-S_{11}$ (without stub) $\rightarrow-S_{11}$ (with stub)
$-\circ-S_{21}$ (without stub) $\rightarrow-S_{21}$ (with stub)

(a)

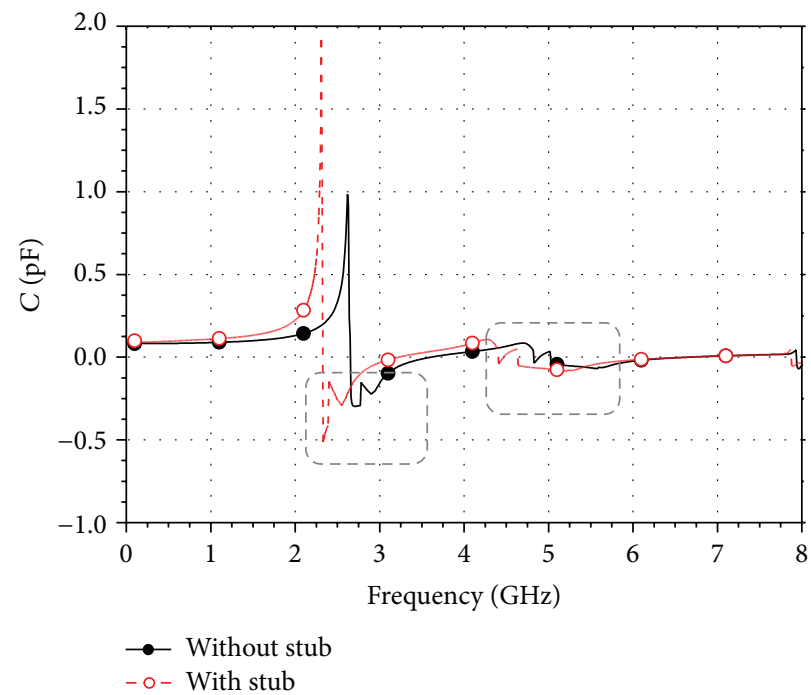

(c)

(b)

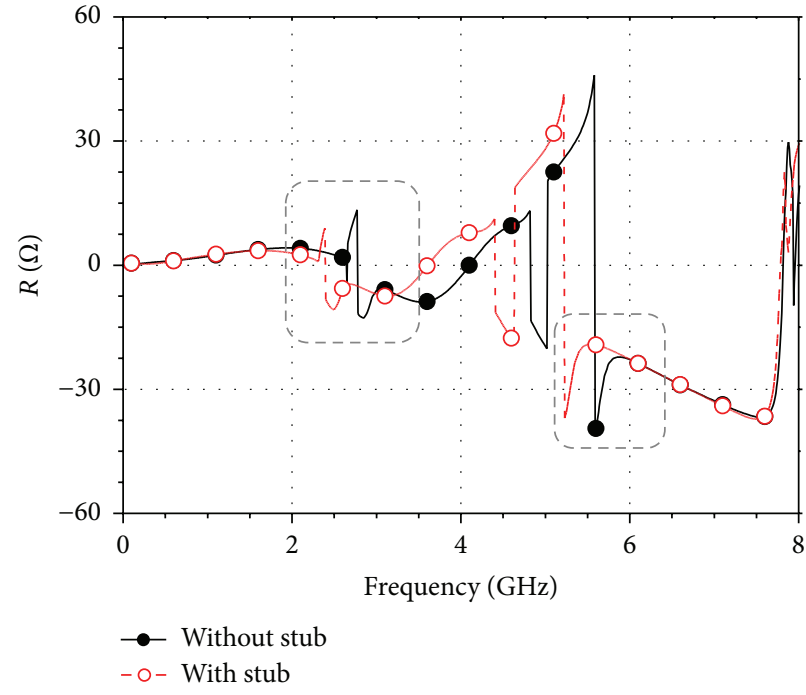

(d)

FIGURE 2: Effect of a T-shaped stub-load on the S-parameters of the proposed DBBSF and its lumped parameters: (a) comparison of the simulated $S$-parameters, (b) net lumped resistance, (c) net lumped inductance, and (d) net lumped capacitance.

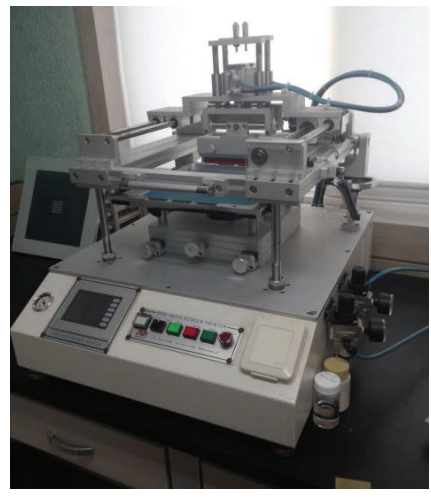

(a)

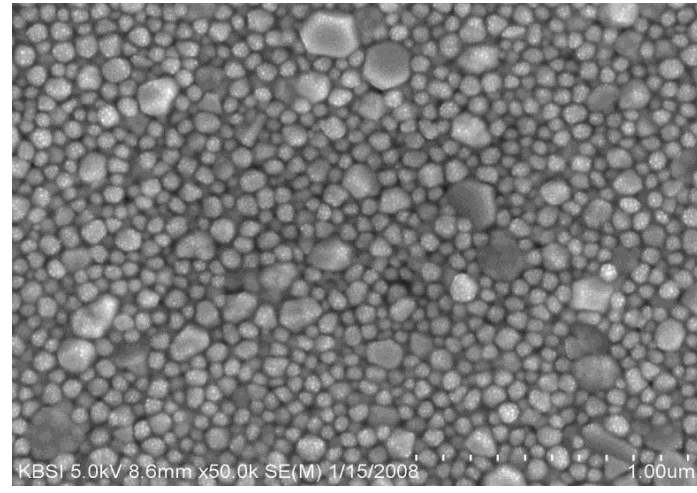

(b)

Figure 3: (a) Screen printer SM-S320 model Sun Mechanix and (b) SEM image of silver nanoparticles used in formulating printing ink. 
The use of stub-loads resulted in DBBSF with central frequencies of 2.55 and $5.3 \mathrm{GHz}$, each of which was shifted downward by $0.35 \mathrm{GHz}$ with respect to the resonant frequencies of the UIRs. To evaluate the effect of the T-shaped stub-load on the central frequencies and return loss, the net lumped element parameters for a propagation distance of $10 \mathrm{~mm}$ were calculated from the simulated $S$-parameters for UIRs and DBBSF [17]. The calculated net lumped parameters, which are displayed in Figure 2, indicated that the use of stub-loads resulted in a significant increase in the net lumped inductance from 0.896 to $0.995 \mathrm{nH}$ and 1.183 to $1.501 \mathrm{nH}$ at the central frequencies of 2.9 and $5.65 \mathrm{GHz}$, respectively, as shown in Figure 2(b). Similarly, the net lumped capacitance increased from 0.055 to $0.224 \mathrm{pF}$ and 0.034 to $0.061 \mathrm{pF}$ at 2.9 and $5.65 \mathrm{GHz}$, respectively, as shown in Figure 2(c). Therefore, the increase in net lumped inductance and capacitance shifted the central frequencies $\left(f=1 / 2 \pi(L C)^{1 / 2}\right)$ downward and noticeably increased the return losses. Additionally, Figure 2(d) shows that the net lumped resistance exhibited peak values, whose magnitude and frequency decreased and shifted downward, respectively, due to use of the stub-loads.

SM-S320 model Sun Mechanix screen printer system, as shown in Figure 3(a), with 250-mesh screen was used to print the Ag conductive lines onto a roll of polyethylene terephthalate (PET) film, which has a width of $200 \mathrm{~mm}$ and a thickness of $250 \mu \mathrm{m}$ and was purchased from SKC Korea. The PET substrate is suitable for screen-printing due to its excellent flexibility and durability. Ag-nanoparticle-based conducting ink (PG-007 BB type, Paru Co., Korea) was used during printing. Because this ink contained $62 \mathrm{wt} \%$ of silver nanoparticles that were 20 to $200 \mathrm{~nm}$ in diameter, as shown in the SEM image displayed in Figure 2(b), the viscosity and surface tension of the silver inks required tuning through a simple ink formulation process. We first formulated the conductive inks to meet the wetting requirements on the PET substrate; then, we adjusted the viscosities by raising the silver-nanoparticle content to $75 \mathrm{wt} \%$. The viscosity of the formulated ink was evaluated using a viscometer (SV-10 Vibro Viscometer, A\&D Co., Japan). Additionally, the surface tension was measured using DCAT 21 (Dataphysics Co., Germany). Ethylene glycol (Aldrich) was used to formulate the Ag ink to achieve a viscosity of $15,000 \mathrm{cp}$ and a surface tension of $47 \mathrm{mN} / \mathrm{m}$. The ethylene glycol stabilized the metal nanoparticles using its ether group, which contains unshared electron pair, to allow the stable use of high concentrations of metal nanoparticles and to increase the viscosity of the solution. A higher viscosity in the printing ink is preferred to obtain a lower resistivity in the printed conductive films with optimized widths. The web transfer speed was set to $5 \mathrm{~m} / \mathrm{min}$ under a roll pressure of $2.5 \mathrm{MPa}$. The printed silver layer was cured for $10 \mathrm{~min}$ by passing through an oven of $150^{\circ} \mathrm{C}$ without additional treatments. To make the back coating a ground conductive layer, we used same ink.

\section{Results and Discussions}

3.1. Characterization of the Printed Ag Lines. Ag-nanoparticle-based, screen-printed conductive films on PET substrates were characterized by XRD to study their crystallinity and structural characteristics. The XRD pattern, which is shown in Figure 4(a), shows a high intensity peak corresponding to the PET substrate at $2 \theta$ angle of $38.1^{\circ}$; this agrees with that reported in the literature $[18,19]$. The presence of peaks at the $2 \theta$ values of $38.4^{\circ}, 44.09^{\circ}$, and $64.36^{\circ}$ corresponds to the (111), (200), and (220) planes of silver, respectively. Thus, the XRD spectrum confirmed the crystalline structure of the silver nanoparticles. No peaks of other impurity crystalline phases were detected, and all peaks in the XRD pattern can be readily indexed to the face-centered cubic structure of silver as per available literature (JCPDS, file number 4-0783).

SEM image of a cross section of a printed Ag line with a width $613 \mu \mathrm{m}$ on PET substrate with a thickness of $250 \mu \mathrm{m}$ is shown in Figure 4(c). A magnified SEM image of a cross section of a printed Ag line, which is depicted in Figure 4(d), shows the thickness results: the middle of the printed line was $12.2 \mu \mathrm{m}$ thick; however, the line thickness marginally decreased to $11.6 \mu \mathrm{m}$ near the edge. This marginal change of the line thickness from center to the edge of the line is expected due to the sintering process of the conductive phase [20]. Based on a four-point measurement technique [21], the conductivity $(\sigma)$ of the printed silver ink after sintering was found to be $2 \times 10^{7} \mathrm{~S} / \mathrm{m}$. Compared with pure silver, the measured $\sigma$ of the printed line was lower; however, $\sigma$ was noticeably higher than previously reported values [22-24] and sufficiently high for practical microwave filter applications. The skin depth $(\delta)$, which is shown in Figure 4(b) for the entire operating frequency band of the proposed DBBSF, was calculated using the following equation [21]:

$$
\delta=\sqrt{\frac{2}{\omega \mu \sigma}}
$$

where $\mu$ indicates the permeability of the printed line and equals 1 and $\omega$ is the angular frequency in $\mathrm{rad} / \mathrm{sec}$. The results indicated that $\delta$ was significantly lower than the measured thickness of the printed line at higher frequencies (e.g., from 2 to $8 \mathrm{GHz}$ ). For lower frequencies (e.g., below $0.5 \mathrm{GHz}$ ), the printed line thickness was marginally higher than $\delta$. Figure 5(a) shows optical microscopy images of the printed lines representing the stub-loads of the proposed DBBSF. The images show that the printed lines were continuous with no voids. However, the center of the lines appeared thicker than the edges; these results agree with the thickness results found by the SEM images and can be caused by a quick viscosity recovery that prevented the ink from completely flattening during drying $[23,24]$. Additionally, the thickness results for the stub-load show a width of $456 \mu \mathrm{m}$, which is $4 \mu \mathrm{m}$ less than the optimized theoretical width indicated in the layout design. This difference in width may be attributed to the tape shrinkage and network compactness after densification by sintering. A decreasing height slope was observed near both edges of the line at a width of $35 \mu \mathrm{m}$. The resulting printed pattern was profiled using atomic force microscopy (AFM), and the corresponding images are displayed in Figure 5(b). The surface roughness results indicate a smooth surface with roughness peaks of $18.64 \mathrm{~nm}$ to $44.79 \mathrm{~nm}$. The surfaces were 


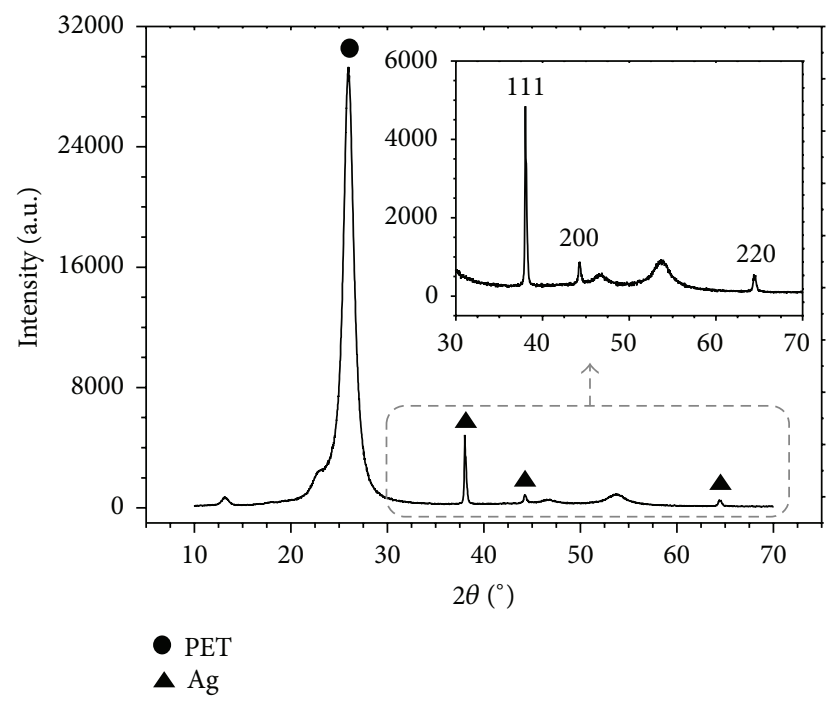

(a)

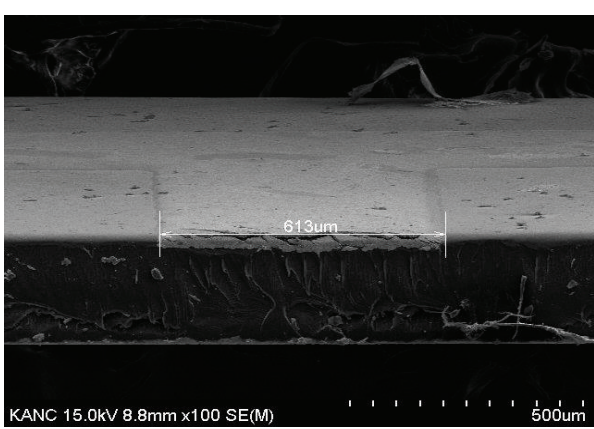

(c)

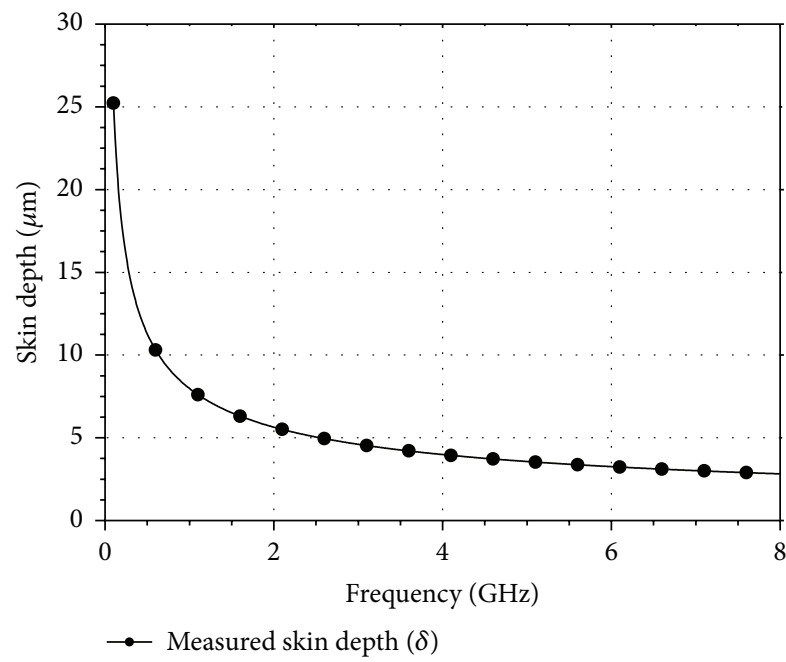

(b)

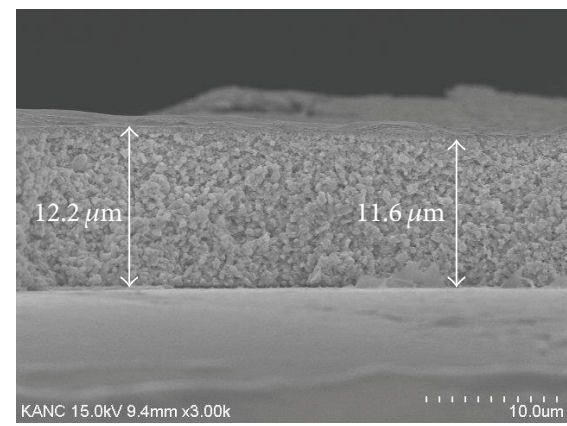

(d)

FIGURE 4: Characterization of the printed Ag films after sintering to achieve the proposed DBBSF: (a) XRD pattern of the screen-printed Ag film on the PET substrate, (b) calculated skin depth $(\delta)$ from the measured conductivity $(\sigma)$, (c) SEM image of a cross section of the screen-printed Ag film on the PET substrate, and (d) magnified SEM image to measure the thickness of the screen-printed Ag film.

shown to have a root mean square (RMS) surface roughness of $7.986 \mathrm{~nm}$.

3.2. Measurement Results of the Printed DBBSF. The screenprinted DBBSF, whose photographs in both normal and bent position are shown in Figure 6, was connected with port connectors using Ag paste to perform electrical measurements. The central frequencies of the fabricated DBBSF that were tested and characterized using Agilent $8510 \mathrm{C}$ vector network analyzer (VNA) were 2.55 and $5.3 \mathrm{GHz}$, each of which was shifted upward by $10 \mathrm{MHz}$ with respect to the simulation results. This shift in frequency may be attributed to the slight difference between fabricated conductive line dimensions compared with the theoretical dimensions; the dielectric loss of the PET substrate; and the dispersion loss at the bends of the UIRs. The return and insertion losses of the first and second stop band were measured to be 27.4 and $28.9 \mathrm{~dB}$ and 1.17 and $1.58 \mathrm{~dB}$, respectively. The measured maximum insertion loss and minimum return loss of 0.68 and $14.5 \mathrm{~dB}$, respectively, for all of the pass bands between the stop bands indicated the excellent out-of-band selectivity of the fabricated DBBSF. The 3-dB fractional bandwidths of the first and second stop bands were measured to be 1.8 and $3.09 \mathrm{GHz}$, respectively.

Table 1, which displays the comparison of the performance of the proposed Ag-nanoparticle-based screenprinted bandstop filter with recently reported several inkjetprinted bandpass filters, indicates that our printed filter exhibits significantly lower insertion losses because of its geometrical structure and relatively higher conductivity printed Ag lines. Additionally, the present DBBSF exhibits higher number of operation bands with large return losses. Moreover, the proposed DBBSF has a compact size of $92.8 \mathrm{~mm}^{2}$.

\section{Conclusion}

In this study, we created an environmentally friendly, highperformance DBBSF based on shunt-connected, T-shaped stub-loaded UIRs. The proposed DBBSF was screen-printed on a low weight, cost-effective PET substrate via screenprinting. Highly conductive $\left(\sigma=2 \times 10^{7} \mathrm{~S} / \mathrm{m}\right)$ lines with favorable characteristics such as uniformity and smooth 

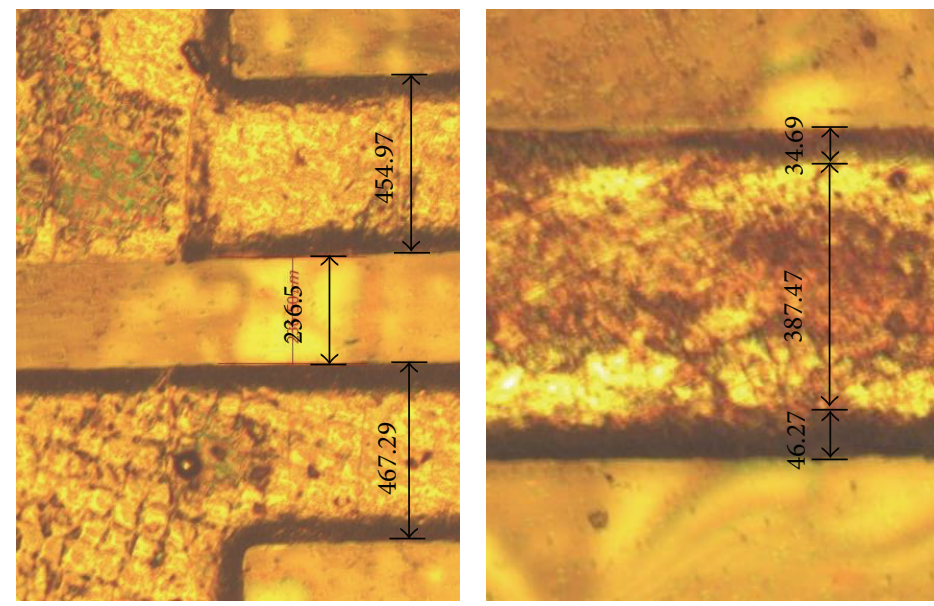

Note: all the dimensions are in $\mu$ m unless unspecified

(a)
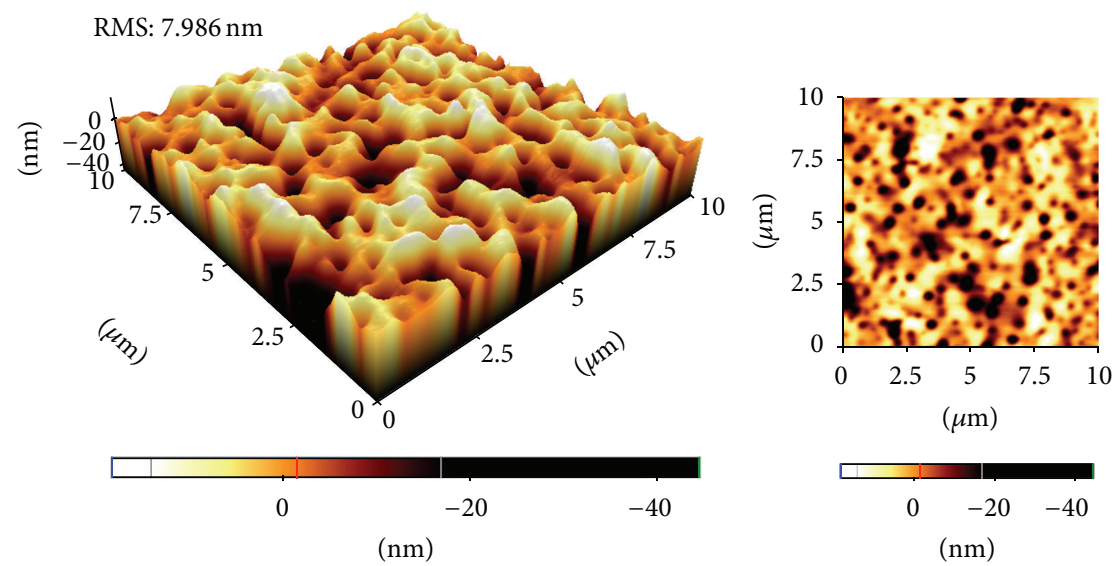

(b)

FiguRE 5: Characterization of the screen-printed line widths and surface morphologies: (a) optical microscopy image of printed stub-loads of the proposed DBBSF. (b) 3D and 2D surface profile using AFM.

TABLE 1: Comparison of the performance of the proposed screen-printed filter with previously reported printed filters.

\begin{tabular}{llcc}
\hline S. number & State-of-the-art in printed microwave filters & $\begin{array}{c}f_{c}(\mathrm{GHz}) \\
{[\mathrm{IL}(\mathrm{dB}) / \mathrm{RL}(\mathrm{dB})]}\end{array}$ & $\begin{array}{c}\text { Film conductivity }(\sigma) \\
(\text { Siemens/m) }\end{array}$ \\
\hline \multirow{2}{*}{ This work } & $\begin{array}{l}\text { T-shaped stub-loaded UIRs based DBBSF using } \\
\text { Ag-nanoparticle-based screen-printing on PET substrate }\end{array}$ & $\begin{array}{r}2.55[1.17 / 27.4], \\
5.3[1.58 / 28.9]\end{array}$ & $2 \times 10^{7}$ \\
\hline Reference [11] & $\begin{array}{l}\text { Hairpin bandpass filter using Ag-nanoparticle-based } \\
\text { inkjet-printing on liquid crystal polymer (LCP) substrate }\end{array}$ & $30[2.41 / 18.9]$ & $2 \times 10^{7}$ \\
\hline Reference [12] & $\begin{array}{l}\text { Interdigital coupled line based bandpass filter using } \\
\text { Ag-nanoparticle-based inkjet-printing LCP substrate }\end{array}$ & $25.7[3.7 / 24]$ & $1 \times 10^{7}$ \\
\hline Reference [13] & $\begin{array}{l}\text { DBR bandpass filter using Ag-nanoparticle-based } \\
\text { inkjet-printing on LCP substrate }\end{array}$ & $5.4[2.18 / 27.8]$ & $4 \times 10^{6}$ \\
\hline \multirow{2}{*}{ Reference [25] } & $\begin{array}{l}\text { Suspended microstrip lines based bandpass filter using } \\
\text { Ag-nanoparticle-based inkjet-printing on dupont kapton HN } \\
\text { polyimide substrate }\end{array}$ & $17[3.6 / 40]$ & $1.5 \times 10^{7}$ \\
\hline
\end{tabular}

S. number = serial number; $f_{c}=$ central frequency; $\mathrm{IL}=$ insertion loss; $\mathrm{RL}=$ return loss. 


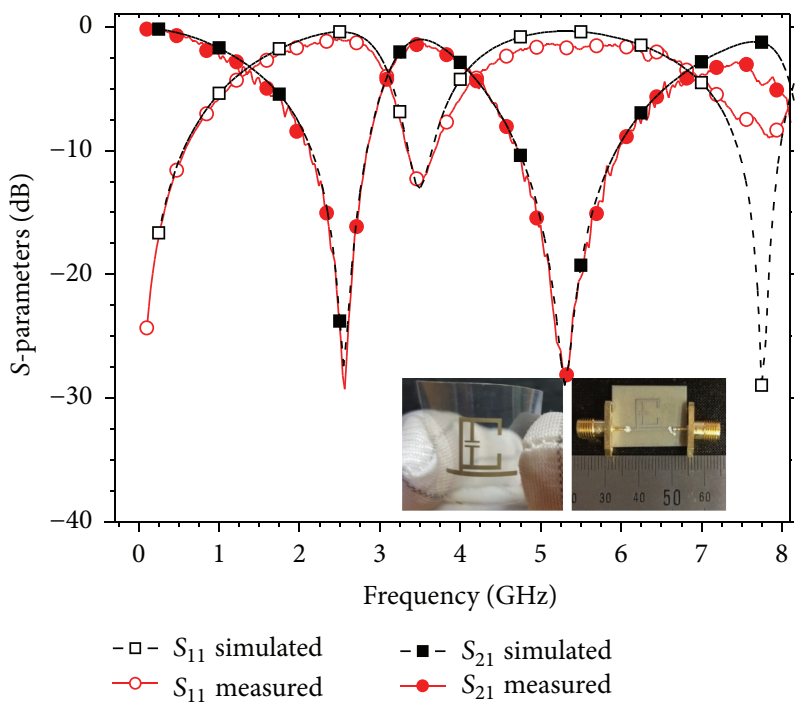

FIGURE 6: Comparison of the simulated and measured $S$-parameters of the proposed screen-printed DBBSF on PET substrate and photographs of printed DBBSF in bent position with connectors.

surfaces were successfully printed using highly viscous Ag conductive paste. The agreement of the simulated and measured results of DBBSF with a compact size of $10 \times 9.28 \mathrm{~mm}^{2}$, measured central frequencies of 2.56 and $5.29 \mathrm{GHz}$, and excellent in-band and out-of-band selectivity indicated that silver-nanoparticle-based screen-printing can be used to successfully fabricate high-performance microwave filters.

\section{Conflict of Interests}

The authors declare that there is no conflict of interests regarding the publication of this paper.

\section{Acknowledgments}

This research was supported by the Basic Science Research Program through the National Research Foundation (NRF) of Korea funded by the Ministry of Science, ICT and Future Planning (no. 2011-0030079) and a grant support from the Korean government (MEST) no. 2012R1A1A2004366. This work was also supported by a Research Grant of Kwangwoon University in 2015. The authors also appreciate the financial support provided by the Ministry of Education of Korea through the Basic Science Research Program at Sunchon National University and by the Global Leading Technology Program of the Office of Strategic R\&D Planning (OSP) through the Ministry of Commerce, Industry and Energy, Republic of Korea (10042537, Printed Electronics Total Solution Development).

\section{References}

[1] W. Wu, L. Liu, Z. Dai et al., "Low-cost, disposable, flexible and highly reproducible screen printed SERS substrates for the detection of various chemicals," Scientific Reports, vol. 5, Article ID 10208, 2015.
[2] A. Huebler, W. Beier, N. Lasch, and T. Fischer, "High volume printing technologies for the production of polymer electronic structures," in Proceedings of the 2nd International Conference on Polymers and Adhesives in Microelectronics and Photonics (POLYTRONIC '02), pp. 172-176, Portland, Ore, USA, June 2002.

[3] W.-Y. Liao and T.-C. Chou, "Fabrication of a planar-form screen-printed solid electrolyte modified $\mathrm{Ag} / \mathrm{AgCl}$ reference electrode for application in a potentiometric biosensor," Analytical Chemistry, vol. 78, no. 12, pp. 4219-4223, 2006.

[4] V. Shanmugam, J. Wong, I. M. Peters et al., "Analysis of fine-line screen and stencil-printed metal contacts for silicon wafer solar cells," IEEE Journal of Photovoltaics, vol. 5, no. 2, pp. 525-533, 2015.

[5] D. Zhou, W. Wu, H. Wang, Y. Jiang, and X. Yao, "The two element antennas using $\mathrm{BiNbO}_{4}$ ceramics as the substrate," Materials Science and Engineering A, vol. 460-461, pp. 652-655, 2007.

[6] W. Cheng, E. J. E. Stuart, K. Tschulik, J. T. Cullen, and R. G. Compton, "A disposable sticky electrode for the detection of commercial silver NPs in seawater," Nanotechnology, vol. 24, no. 50, Article ID 505501, 2013.

[7] J. Virkki, T. Björninen, S. Merilampi, L. Sydänheimo, and L. Ukkonen, "Manufacturing and applications of screen-printed RFID tags on paper substrate," in Proceedings of the Progress in Electromagnetics Research Symposium (PIERS '13), pp. 562-566, Stockholm, Sweden, August 2013.

[8] W. Wu, S. Yang, S. Zhang, H. Zhang, and C. Jiang, "Fabrication, characterization and screen printing of conductive ink based on carbon@Ag core-shell nanoparticles," Journal of Colloid and Interface Science, vol. 427, pp. 15-19, 2014.

[9] H. Wu, S. W. Chiang, W. Lin et al., "Towards practical application of paper based printed circuits: capillarity effectively enhances conductivity of the thermoplastic electrically conductive adhesives," Scientific Reports, vol. 4, article 6275, 2014.

[10] J. Virkki, T. Björninen, T. Kellomäki et al., "Reliability of washable wearable screen printed UHF RFID tags," Microelectronics Reliability, vol. 54, no. 4, pp. 840-846, 2014.

[11] H.-L. Kao, C.-L. Cho, X. Dai et al., "Hairpin bandpass filter on liquid crystal polymer substrate using inkjet printing technology," in Proceedings of the IEEE MTT-S International Symposium Digest (IMS '13), pp. 1-4, Seattle, Wash, USA, June 2013.

[12] H.-L. Kao, C.-L. Cho, and L.-C. Chang, "Inkjet-printed interdigital coupled line filter on liquid crystal polymer substrate," IEEE Electron Device Letters, vol. 34, no. 12, pp. 1584-1586, 2013.

[13] H.-L. Kao, C.-L. Cho, L.-C. Chang, C.-S. Yeh, B.-W. Wang, and H.-C. Chiu, "Inkjet printing RF bandpass filters on liquid crystal polymer substrates," Thin Solid Films, vol. 544, pp. 64-68, 2013.

[14] F.-C. Chen, J.-M. Qiu, and Q.-X. Chu, "Dual-band bandstop filter using stub-loaded resonators with sharp rejection characteristic," Electronics Letters, vol. 49, no. 5, pp. 351-353, 2013.

[15] K. K. Adhikari and N. Y. Kim, "A miniaturized quadband bandstop filter with high selectivity based on shuntconnected, T-shaped stub-loaded, stepped-impedance resonators," Microwave and Optical Technology Letters, vol. 57, no. 5, pp. 1129-1132, 2015.

[16] K. K. Adhikari and N.-Y. Kim, "Microstrip triband bandstop fitler with sharp stop band skirts and independently controllable second stop band response," The Scientific World Journal, vol. 2014, Article ID 760838, 9 pages, 2014. 
[17] W. K. Kim, Y. M. Jung, J. H. Cho et al., "Radio-frequency characteristics of graphene oxide," Applied Physics Letters, vol. 97, no. 19, Article ID 193103, 2010.

[18] M. G. Faraj, K. Ibrahim, and M. H. Eisa, "Investigation of the optical and structural properties of thermally evaporated cadmium sulphide thin films on polyethylene terephthalate substrate," Materials Science in Semiconductor Processing, vol. 14, no. 2, pp. 146-150, 2011.

[19] A. N. Banerjee, C. K. Ghosh, K. K. Chattopadhyay et al., "Lowtemperature deposition of $\mathrm{ZnO}$ thin films on PET and glass substrates by DC-sputtering technique," Thin Solid Films, vol. 496, no. 1, pp. 112-116, 2006.

[20] R. Faddoul, N. Reverdy-Bruas, and A. Blayo, "Formulation and screen printing of water based conductive flake silver pastes onto green ceramic tapes for electronic applications," Materials Science and Engineering: B, vol. 177, no. 13, pp. 1053-1066, 2012.

[21] K. Park, D. Seo, and J. Lee, "Conductivity of silver paste prepared from nanoparticles," Colloids and Surfaces A: Physicochemical and Engineering Aspects, vol. 313-314, pp. 351-354, 2008.

[22] M. Lahti and V. Lantto, "Passive RF band-pass filters in an LTCC module made by fine-line thick-film pastes," Journal of the European Ceramic Society, vol. 21, no. 10-11, pp. 1997-2000, 2001.

[23] R. Faddoul, N. Reverdy-Bruas, and J. Bourel, "Silver content effect on rheological and electrical properties of silver pastes," Journal of Materials Science: Materials in Electronics, vol. 23, no. 7, pp. 1415-1426, 2012.

[24] L. B. Wang, K. Y. See, J. W. Zhang, B. Salam, and A. C. W. $\mathrm{Lu}$, "Ultrathin and flexible screen-printed metasurfaces for EMI shielding applications," IEEE Transactions on Electromagnetic Compatibility, vol. 53, no. 3, pp. 700-705, 2011.

[25] D. Sette, A. Blayo, C. Poulain, and D. Mercier, "Silver nanoparticle inkjet printed $17 \mathrm{GHz}$ filter," in Proceedings of the IEEE MTTS International Microwave Symposium Digest (IMS '13), pp. 1-4, Seattle, Wash, USA, June 2013. 

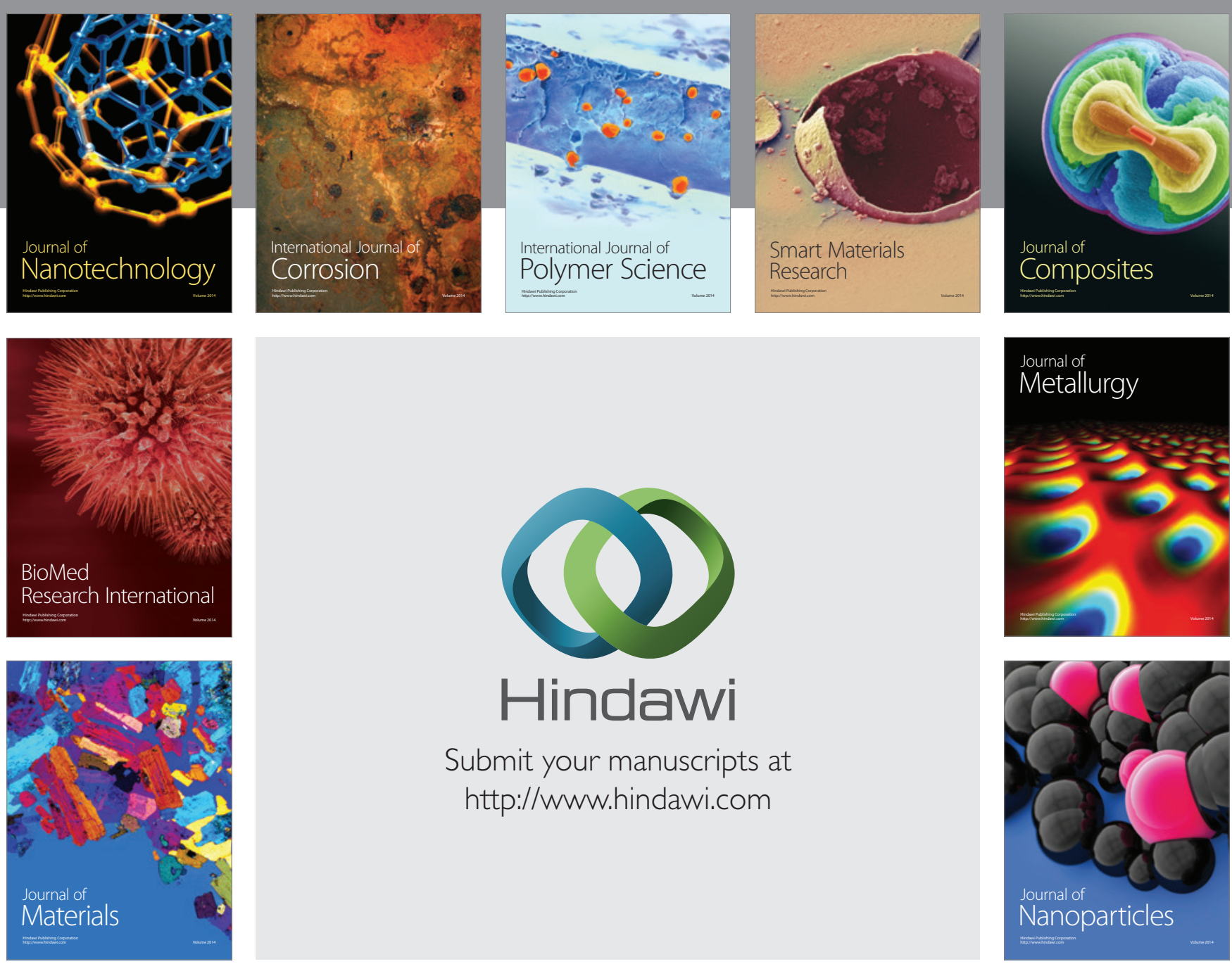

Submit your manuscripts at http://www.hindawi.com
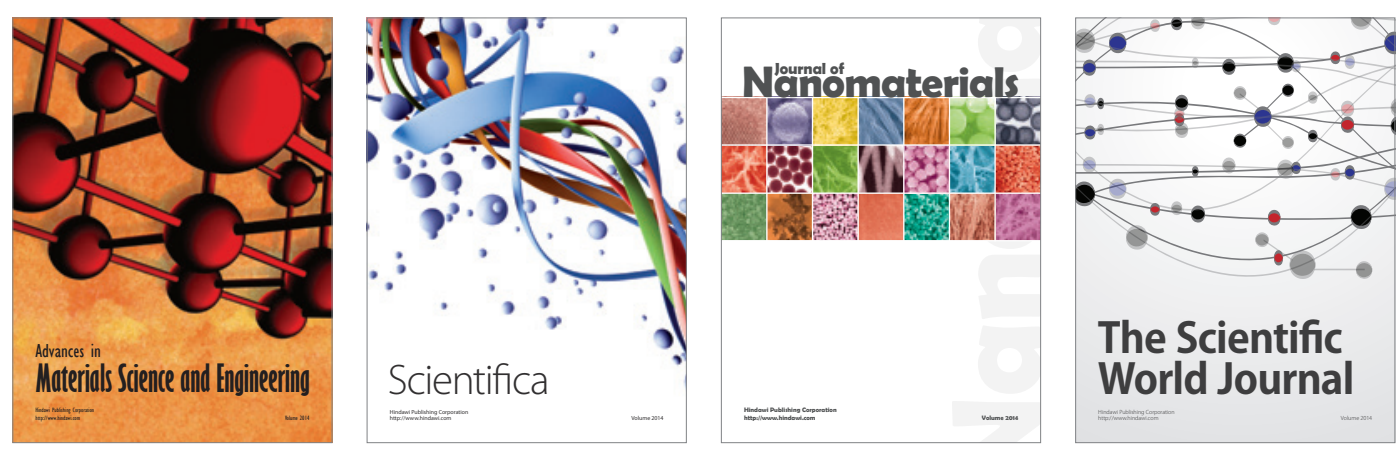

\section{The Scientific World Journal}
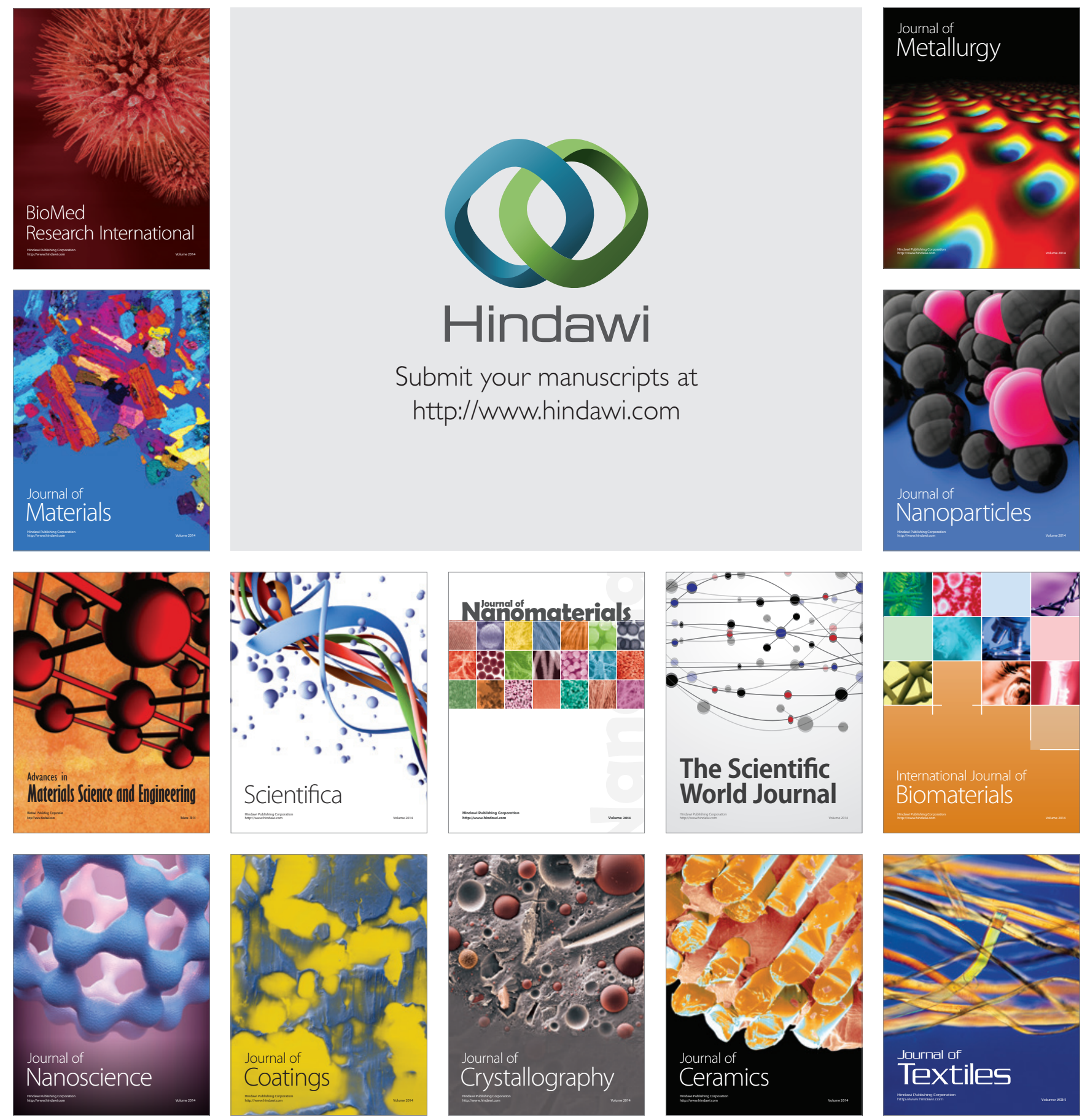